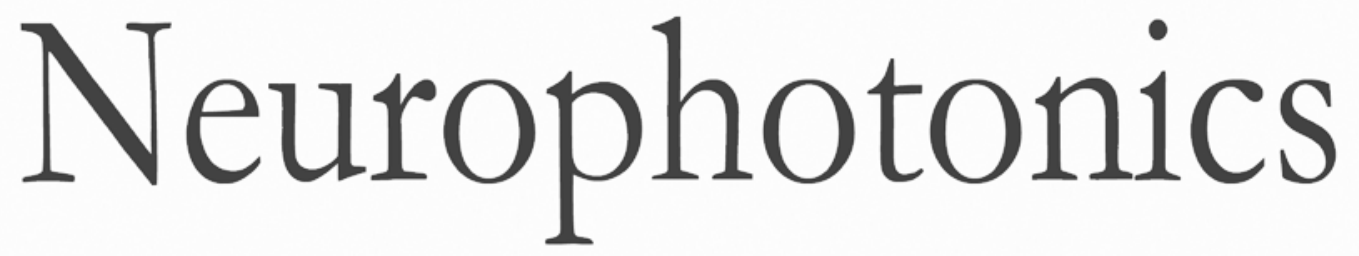

\title{
Action potential block in neurons by infrared light
}

\author{
Alex J. Walsh \\ Gleb P. Tolstykh \\ Stacey Martens \\ Bennett L. Ibey \\ Hope T. Beier
}




\section{Action potential block in neurons by infrared light}

\author{
Alex J. Walsh, ${ }^{\text {a,b }}$ Gleb P. Tolstykh, ${ }^{\text {c }}$ Stacey Martens, ${ }^{b}$ \\ Bennett L. Ibey, ${ }^{b}$ and Hope T. Beier ${ }^{b, *}$ \\ ${ }^{a}$ National Research Council, JBSA Fort Sam Houston, Texas 78234 \\ United States \\ ${ }^{b}$ Air Force Research Laboratory, Bioeffects Division, \\ JBSA Fort Sam Houston, Texas 78234, United States \\ ${ }^{\circ}$ General Dynamics Information Technology, JBSA Fort Sam \\ Houston, Texas 78234, United States
}

Abstract. Short infrared laser pulses (SILP) have many physiological effects on cells, including the ability to stimulate action potentials (APs) in neurons. Here, we show that SILPs can also reversibly block APs. Reversible AP block in hippocampal neurons was observed following SILP $\left(0.26\right.$ to $0.96 \mathrm{~J} / \mathrm{cm}^{2} ; 1.37$ to $\left.5.01 \mathrm{~ms} ; 1869 \mathrm{~nm}\right)$ with the block persisting for more than $1 \mathrm{~s}$ with exposures greater than $0.69 \mathrm{~J} / \mathrm{cm}^{2}$. AP block was sustained for $30 \mathrm{~s}$ with SILPs pulsed at 1 to $7 \mathrm{~Hz}$. Full recovery of neuronal activity was observed 5 to $30 \mathrm{~s}$ post SILP exposure. These results indicate that SILP can be used for noncontact, reversible AP block. Due to the high spatial precision and noncontact manner of infrared light delivery, AP block by SILP (infrared neural inhibition) has the potential to transform medical care for sustained pain inhibition and suppression of unwanted nerve activity. (- 2016 Society of Photo-Optical Instrumentation Engineers (SPIE) [DOI: 10.1117/1.NPh.3.4.040501]

Keywords: optogenetics; neurons; action potential; infrared light/radiation; fast thermal gradient.

Paper 16043LRR received Jul. 1, 2016; accepted for publication Nov. 8, 2016; published online Dec. 1, 2016.

Short infrared laser pulses (SILPs) are pulses of infrared light at wavelengths between 1400 and $2100 \mathrm{~nm}$ and durations of micro- to milliseconds. SILPs have been observed to induce action potentials (APs) in neurons, a phenomenon termed infrared neural stimulation (INS). ${ }^{1-5}$ While the mechanisms behind INS are not fully understood, it is dependent on the fast thermal gradient induced by the infrared exposure ${ }^{5}$ and is characterized by rapid increase in intracellular calcium. ${ }^{6,7}$ Preliminary reports have demonstrated that SILPs may also block APs; ${ }^{4,8,9}$ however, these reports measured reduced electrical activity in neuron networks downstream of repeated SILP exposures and may report indirect effects of INS on inhibitory neurons. Additionally, infrared radiation may cause AP block directly, which we term infrared neural inhibition (INI).

AP block can be achieved by electrical, pharmacological, and optogenetic methods. However, electrical block requires either invasive and direct-contact electrodes or suffers from nonspecificity. ${ }^{10,11}$ Pharmacological means of AP block suffer from nonspecificity and potential off-target effects. ${ }^{12}$ Optogenetic means of AP block, such as by halorhodopsins, light-activated chloride ion channels, require genetic manipulation of the target cells. $^{13,14}$ To date, a noninvasive, nongenetic, highly specific, and reversible method for AP block has remained elusive. Such a method could be transformative for overcoming present limitations in electrically based neural interfaces of prosthetics and in medical devices to control unwanted APs.

Robust study of AP block requires repeated and precise AP stimulation and membrane voltage recording. Electrical means of stimulation and recording can be performed by patch clamp electrophysiology. However, SILPs have been found to reduce pipet and seal resistance, leading to confounded membrane voltage measurements. ${ }^{15,16}$ Therefore, we applied an optogenetic approach for noninvasive/noncontact and reliable AP stimulation and recording.

We used the Optopatch2 plasmid, which consists of a bluelight activated channel rhodopsin, CheRiff, and a red-light fluorescent archaerhodopsin 3 (Arch) protein, QuasAr2, that allows fluorescence imaging of membrane potential ${ }^{17,18}$ (Fig. 1). All procedures were performed in compliance with Institutional Animal Care and Use Committee protocols and meet the US National Institutes of Health Guide for the care and use of laboratory animals. Embryonic (E18) rat hippocampi were obtained from BrainBits, LLC. Hippocampi were incubated in papain ( $2 \mathrm{mg} / \mathrm{ml}$ ) for $10 \mathrm{~min}$ and dissociated into a single cell suspension. Neurons were immediately transfected with Optopatch2 (Addgene 51694) ${ }^{17}$ by an Amaxanucleofector device using standard protocol. Transfected neurons were plated at a density of $\sim 10,000$ cells $/ \mathrm{cm}^{2}$ on glass bottom dishes (MatTek). Neurons were cultured in neurobasal-based medium (NBActiv1TM; BrainBits) supplemented with $25-\mu \mathrm{M}$ glutamate for 4 days. Subsequent media exchanges once weekly were with NBActiv4 ${ }^{\mathrm{TM}}$ (BrainBits). Experiments were performed on days 14 to 20 posttransfection. Neurons were incubated with $5 \mu \mathrm{M}$ all-trans retinol for $30 \mathrm{~min}$ prior to imaging, then washed with and imaged in standard outside solution $(2 \mathrm{mM} \mathrm{MgCl}$, $5 \mathrm{mM} \mathrm{KCl}, 10 \mathrm{mM}$ HEPES, $10 \mathrm{mM}$ glucose, $2 \mathrm{mM} \mathrm{CaCl}_{2}$, and $13.5 \mathrm{mM} \mathrm{NaCl}, \mathrm{pH}=7.4$, osmolarilty $=300 \mathrm{mOsm}$ ).

QuasAr2 fluorescence was excited with $6.7 \mathrm{~mW}$ $\left(\sim 9.5 \mathrm{~W} / \mathrm{cm}^{2}\right)$ of $647-\mathrm{nm}$ light from an Argon-Krypton Ion laser through a $40 \times$ oil-immersion objective (1.3 NA) on an inverted Olympus microscope. Emission light was filtered by a 647-nm long pass filter (Semrock) and imaged onto an Andor iXon EMCCD Camera. To achieve a frame rate of $1004 \mathrm{~Hz}$, the field of view was cropped and binned $2 \times 2$ to an image size of $48 \times 48$ pixels. Throughout the infrared exposure experiments, QuasAr2 was continuously imaged. No loss of AP spikes in QuasAr2 fluorescence was observed for lengthy (60 s+) imaging sessions.

To induce APs, transfected cells were illuminated with blue light $\left(55 \mu \mathrm{W}, 78 \mathrm{~mW} / \mathrm{cm}^{2}, 488 \mathrm{~nm}\right.$, Argon laser; 5-ms pulse duration, $10 \mathrm{~Hz}$ ) coaligned with the $647-\mathrm{nm}$ excitation laser into the microscope. An electronic shutter was used to create pulses. Validation of AP induction by blue light and recording of the QuasAr2 fluorescence was verified by a tetrodotoxin (TTX) experiment. TTX inhibited blue-light activated APs (data not shown).

SILPs (Aculight Capella Laser) at $1869 \mathrm{~nm}$ were delivered to the neuron of interest by a $200-\mu \mathrm{m}$ fiber, angled at $45 \mathrm{deg}$ [Fig. 2(a)]. The tip of the fiber was placed $\sim 215 \mu \mathrm{m}$ along the diagonal away from the cell to reduce interference and 
(a)

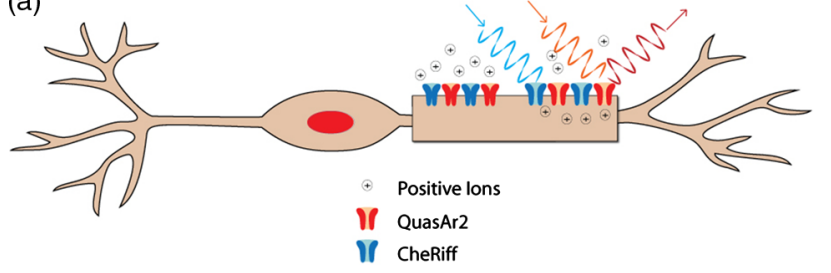

(b)
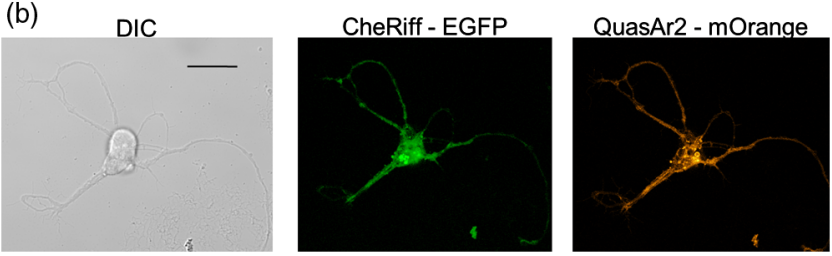

Fig. 1 (a) Drawing of a neuron expressing the Optopatch2 proteins CheRiff and QuasAr2. CheRiff is a cation channel activated by blue light. QuasAr2 is fluorescent and the fluorescence intensity increases with membrane voltage. (b) Representative confocal differential interference contract and fluorescence images of a neuron, scale bar is $50 \mu \mathrm{m}$. Fluorescence image of enhanced green fluorescent protein (EGFP) confirms successful transfection of CheRiffEGFP. Fluorescence image of QuasAr2-mOrange confirms successful transfection of QuasAr2-mOrange.

noise from scattered light from the fiber. The low plating density allowed only a single cell to be illuminated by each SILP to minimize interference from neighboring neurons [Fig. 2(b)]. Infrared irradiance was varied by changing the pulse width. Neurons were exposed to a single SILP. A range of pulse widths and radiant exposures (Table 1) were evaluated and experiments were repeated on different neurons for a total $n$ of 9 to 33 neurons per SILP exposure. Infrared radiant exposures and temperature gradients at the plane of the cells were determined by the scalable effects simulation environment (SESE) model as previously described. ${ }^{6}$ Briefly, the SESE model is a three-dimensional numerical simulation model that combines light transport and heat transfer. The SESE approximation of the laser energy does not account for thermal lensing due to the fast thermal gradient induced by the infrared exposure and may, therefore, underestimate the actual radiant exposure of the cells. The SESE model revealed the duration and magnitude of the IRinduced thermal gradient [Fig. 2(c) and Table 1].

A single SILP blocked APs in neurons (Fig. 3). The average fluorescence for each cell over time was filtered to remove SILP artifact and high-frequency noise and plotted as $\Delta F / F$. Spike peaks were fixed to a constant value to account for under sampling due to camera limitations. Here, firing efficiency refers to
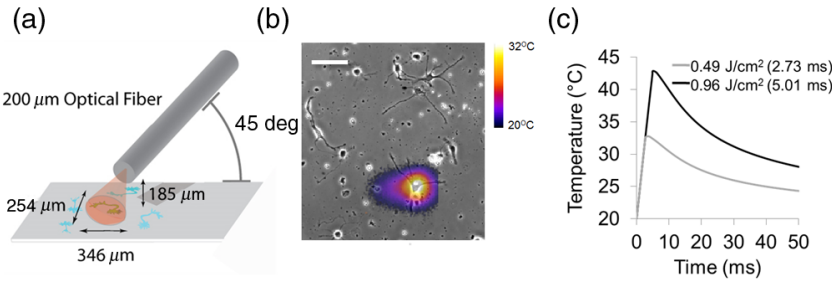

Fig. 2 (a) Placement of the infrared light delivery fiber relative to the neuron. (b) Representative image shows the confluency of the neurons and the peak of the thermal gradient of a $2.73 \mathrm{~ms}\left(0.52 \mathrm{~J} / \mathrm{cm}^{2}\right)$ infrared light pulse. The thermal image was acquired with a FLIR thermal camera. Scale bar is $250 \mu \mathrm{m}$. (c) SILP induced thermal gradient due to 0.52 and $0.96 \mathrm{~J} / \mathrm{cm}^{2}$ exposures.
Table 1 SILP exposures and corresponding temperature gradients.

\begin{tabular}{lcccc}
$\begin{array}{l}\text { Pulse width } \\
(\mathrm{ms})\end{array}$ & $\begin{array}{c}\text { Energy } \\
(\mathrm{mJ})\end{array}$ & $\begin{array}{c}\text { Radiant exposure } \\
\text { at cells }\left(\mathrm{J} / \mathrm{cm}^{2}\right)\end{array}$ & $\Delta T\left({ }^{\circ} \mathrm{C}\right)$ & $\begin{array}{c}n \text { [Figs. 3(g) } \\
\text { and 3(h) }\end{array}$ \\
\hline 0.70 & 0.86 & 0.13 & 3.2 & 18 \\
1.37 & 1.68 & 0.26 & 6.3 & 19 \\
2.15 & 2.64 & 0.41 & 9.9 & 9 \\
2.73 & 3.35 & 0.52 & 12.5 & 26 \\
3.58 & 4.39 & 0.69 & 16.4 & 13 \\
4.10 & 5.03 & 0.79 & 18.8 & 33 \\
5.01 & 6.14 & 0.96 & 23.0 & 10 \\
5.47 & 6.71 & 1.05 & 25.1 & 27 \\
5.73 & 7.03 & 1.10 & 26.3 & 12 \\
\hline
\end{tabular}

percentage of APs observed in response to the blue light stimulus with a maximum of $100 \%$ indicating an AP immediately follows every blue light exposure and $0 \%$ indicating no APs with blue light stimulation. Representative QuasAr2 fluorescence spike plots demonstrate the pre-SILP 100\% AP firing efficiency, the duration of the AP block following 0.52, 0.96, and $1.05 \mathrm{~J} / \mathrm{cm}^{2}$ SILP exposure, and postexposure activity [Figs. 3(a)-3(c)]. Recovery of APs with $100 \%$ firing efficiency was observed following 0.52 and $0.96 \mathrm{~J} / \mathrm{cm}^{2}$ exposures, albeit after a period with lower firing efficiency [Figs. 3(d) and 3(e)]. While APs were observed following the block induced by the $1.05 \mathrm{~J} / \mathrm{cm}^{2}$ SILP, firing efficiency remained around $35 \%$, even 5 min after the SILP, suggesting significant neural damage [Fig. 3(f)]. These results suggest a threshold to recovery of $100 \%$ firing efficiency between 0.96 and $1.05 \mathrm{~J} / \mathrm{cm}^{2}$.

Overall, the length of AP block (complete suppression of AP) and recovery to $100 \%$ firing efficiency correlated with blue light stimulation scaled with SILP irradiance [Figs. 3(g) and 3(h)]. The AP block persisted for $0.26 \mathrm{~s}$ with a $0.26 \mathrm{~J} / \mathrm{cm}^{2}$ exposure $(p<0.0001$ versus $0 \mathrm{~s})$ and as long as $5.37 \mathrm{~s}$ with a $1.10 \mathrm{~J} / \mathrm{cm}^{2}$ exposure $(p<0.0001$ versus $0 \mathrm{~s}$ and $p<0.0001$ versus $\left.0.26 \mathrm{~J} / \mathrm{cm}^{2}\right)$. The length of AP block increased with increased exposure, and followed an exponential trend, AP $\operatorname{block}(\mathrm{s})=0.12 \mathrm{e}^{3.3 * \operatorname{exposure}\left(\mathrm{J} / \mathrm{cm}^{2}\right)}, R^{2}=0.99$. The average time to recovery of $100 \%$ firing efficiency for a neuron following a $0.26 \mathrm{~J} / \mathrm{cm}^{2}$ exposure was $2.75 \mathrm{~s}$, and a maximum average recovery time of $11.1 \mathrm{~s}$ was observed following a $0.96 \mathrm{~J} / \mathrm{cm}^{2}$ exposure [Fig. 3(h)]. For exposures between 0.13 and $0.96 \mathrm{~J} / \mathrm{cm}^{2}$, the recovery time increased linearly with increased exposures, recovery $(\mathrm{s})=12.5 * \operatorname{exposure}\left(\mathrm{J} / \mathrm{cm}^{2}\right)-1.0$ [Fig. 3(h), $R^{2}=0.98$ ], at exposures greater than $0.96 \mathrm{~J} / \mathrm{cm}^{2}$, recovery was not observed within 5 min.

By delivering $30 \mathrm{~s}$ of a SILP train $\left(0.52\right.$ or $0.79 \mathrm{~J} / \mathrm{cm}^{2}$, pulsed at 1 to $7 \mathrm{~Hz}$ ), AP frequency was significantly reduced (Fig. 4). A representative QuasAr2 fluorescence spike plot demonstrates uniform AP generation before SILP exposure, reduction during exposure $\left(0.52 \mathrm{~J} / \mathrm{cm}^{2} ; 3 \mathrm{~Hz}\right)$, and recovery (Figs. 4(a)-4(c)]. Significant reductions in the average number of APs per second were observed during the SILP train: $95.4 \%$ inhibited with $0.96 \mathrm{~J} / \mathrm{cm}^{2}$ at $5 \mathrm{~Hz}$ and $97.4 \%$ inhibited with $0.52 \mathrm{~J} / \mathrm{cm}^{2}$ at $7 \mathrm{~Hz}$ [Figs. 4(d) and 4(e)]. Following 

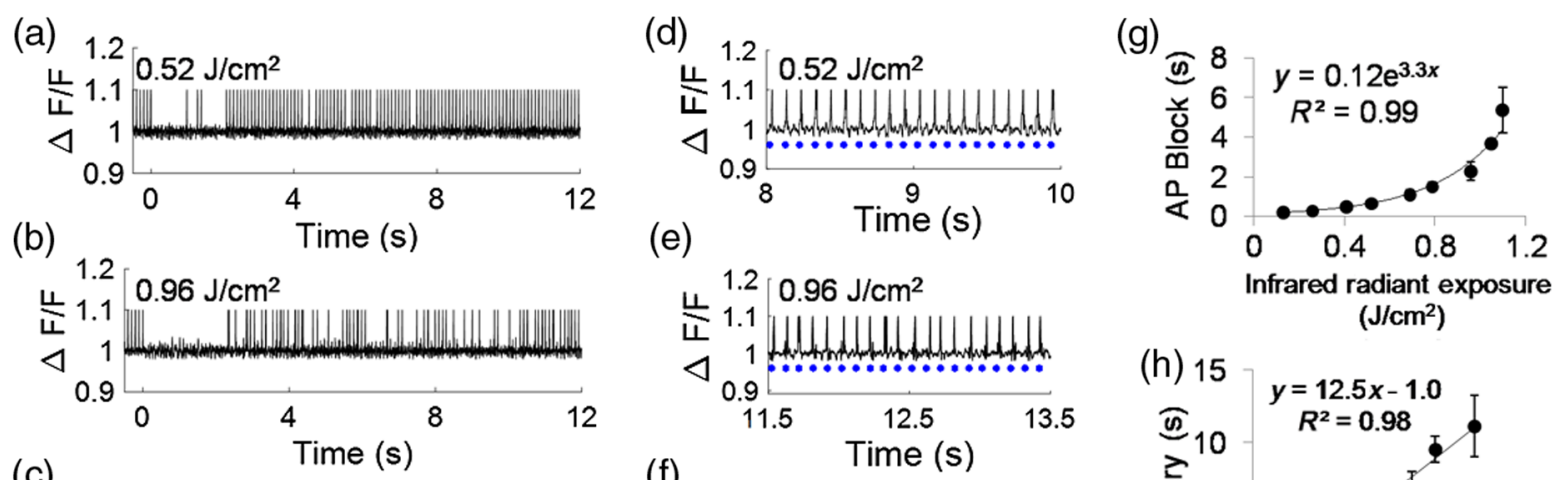

e)

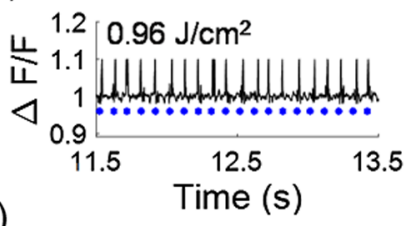

Infrared radiant exposure $\left(\mathrm{J} / \mathrm{cm}^{2}\right)$

(c)

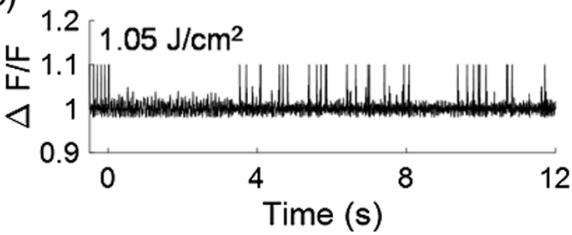

(f)
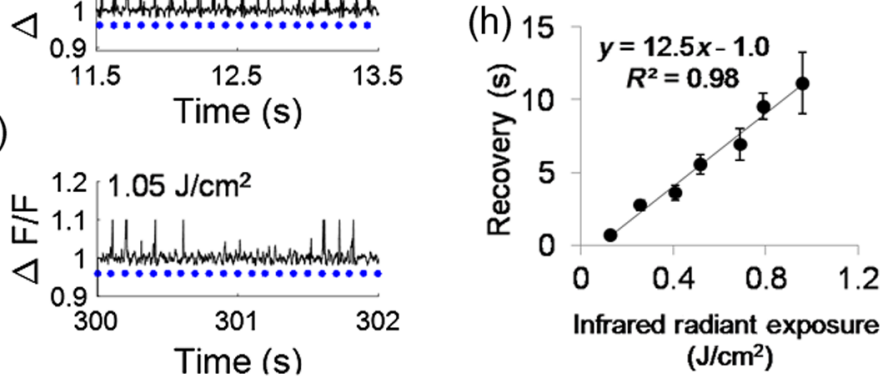

Fig. 3 Representative QuasAr2 fluorescence spike plots (from a single neuron per exposure) show block and recovery following a (a) 0.52 , (b) 0.96 , and (c) $1.05 \mathrm{~J} / \mathrm{cm}^{2}$ SILP. Blue light was pulsed at $10 \mathrm{~Hz}$. SILP exposure at $t=0 \mathrm{~s}$. Recovery of blue light stimulated AP is demonstrated at (d) 8 to $10 \mathrm{~s}$ for the $0.52 \mathrm{~J} / \mathrm{cm}^{2}$ SILP [same cell as (a)], (e) 11.5 to $13.5 \mathrm{~s}$ for the $0.96 \mathrm{~J} / \mathrm{cm}^{2}$ SILP [same cell as (b)], and (f) $300(5 \mathrm{~min})$ to $302 \mathrm{~s}$ for the $1.05 \mathrm{~J} / \mathrm{cm}^{2}$ SILP [same cell as (c)]. Blue dots represent blue light exposure. (g) The average length of SILP-induced AP block increased with increased exposure $(n=9$ to 33 cells/data point; mean \pm SE). (h) The average time of recovery to $100 \%$ firing efficiency increased with SILP exposure ( $n=9$ to 22 cells, mean \pm SE).

(a)

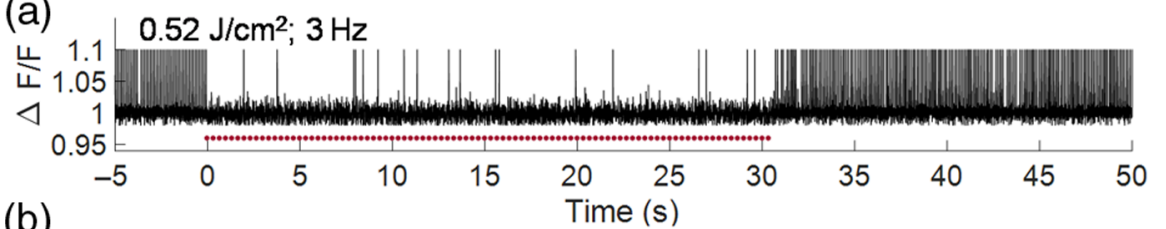

(b)

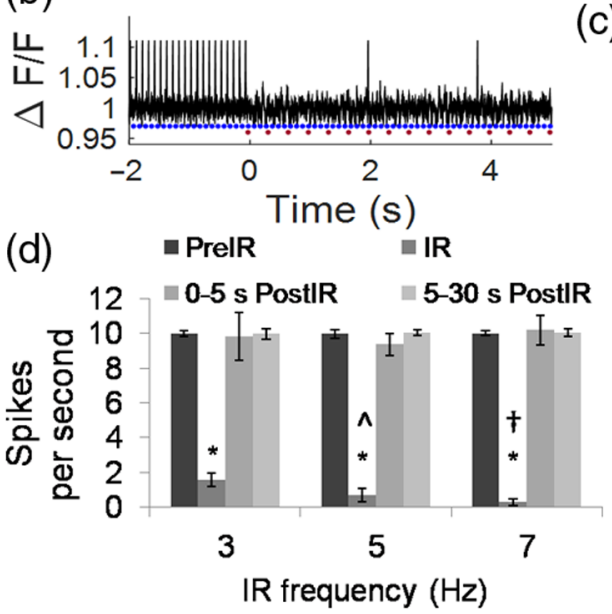

(c)

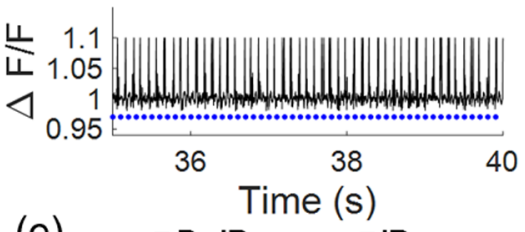

(e)

PrelR $\quad$ IR

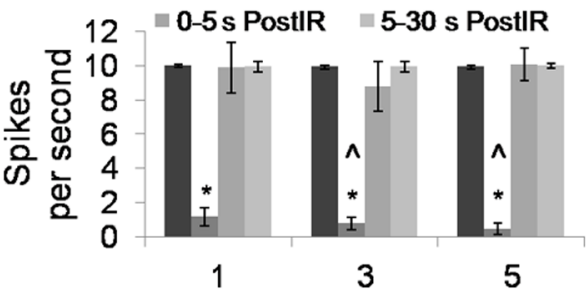

IR frequency $(\mathrm{Hz})$

Fig. 4 (a) Representative QuasAr2 fluorescence spike plot demonstrating consistent AP generation prior to SILPs, reduced AP activity during the repeated SILPs $\left(0.52 \mathrm{~J} / \mathrm{cm}^{2} ; 3 \mathrm{~Hz}\right)$, and following the SILP exposure. Blue light (5-ms exposure) was pulsed at $10 \mathrm{~Hz}$. Red dots represent SILPs. (b) Enlarged display of -2 to $5 \mathrm{~s}$ data in (a), showing the consistent APs prior to the SILP exposure $(t<0)$; and the block during exposure $(t>0)$. Blue dots represent blue light exposure. (c) Enlarged display of the 35 to $40 \mathrm{~s}$ data in (a) showing recovery of consistent AP. (d) Average SPS for neurons prior to SILP (blue led pulsed at $10 \mathrm{~Hz}$ ), during $30 \mathrm{~s}$ of $0.52 \mathrm{~J} / \mathrm{cm}^{2}$ SILP pulsed at $3(n=6$ cells), $5(n=6$ cells), or 7 ( $n=6$ cells) Hz, and following SILP. Mean \pm SE, $n=6$ to 9 cells. ${ }^{*} p<0.05$ versus pre-SILP. ${ }^{\wedge} p<0.05$ versus $3 \mathrm{~Hz}$ SILP. $\dagger p<0.05$ versus $5 \mathrm{~Hz}$ SILP. (e) Average SPS for neurons prior to SILP (blue light pulsed at $10 \mathrm{~Hz}$ ), during $30 \mathrm{~s}$ of $0.79 \mathrm{~J} / \mathrm{cm}^{2}$ SILP pulsed at 1 ( $n=5$ cells), 3 ( $n=7$ cells), or 5 ( $n=6$ cells) Hz, and following SILP. Mean \pm SE, ${ }^{*} p<0.05$ versus pre-SILP. $\wedge p<0.05$ versus infrared $1 \mathrm{~Hz}$. 
SILP-induced AP block, neuronal behavior recovered to ten spikes per second (SPS) (Figs. 4(c) and 4(e)]. A significant reduction $(p<0.001)$ in the SPS was observed with increasing pulse frequency, from 1 to $3 \mathrm{~Hz}$ or $5 \mathrm{~Hz}$ for the $0.79 \mathrm{~J} / \mathrm{cm}^{2}$ exposure and from 3 to $5 \mathrm{~Hz}$ and from 5 to $7 \mathrm{~Hz}$ for the $0.52 \mathrm{~J} / \mathrm{cm}^{2}$ exposure [Figs. 4(d) and 4(e)]. These results indicate that SILP can be used for noncontact and reversible AP block, and the efficiency of the AP block scales with infrared exposure frequency and duration.

Altogether, these results demonstrate that SILP can directly block APs in primary, mammalian neurons. The observed AP block can be attributed to the SILP exposure due to the direct, single cell infrared light exposure and immediacy of block onset following SILP exposure. However, some of the observed activity may be due to synaptic events. SILP has been shown to affect neuronal behavior previously, primarily by stimulating APs, yet the mechanisms behind SILP effects remain unknown. ${ }^{1-4,8}$ Current theories to explain mechanisms for INS and INI include direct change of membrane capacitance by the thermal gradient, ion flow through heat activated or stretch activated transient receptor potential channels, heat inactivation of ion channels, nanopore formation (and subsequent ion flow), and/or stimulated calcium-induced-calcium release. ${ }^{5-8,15,19}$ The observed SILP AP block persists for several seconds at maximum radiant exposures, a length of time well beyond the period of laserinduced heating $(\sim 5 \mathrm{~ms}){ }^{6}$ Previous work has shown indirect evidence of phosphatidylinositol 4,5-bisphosphate $\left(\mathrm{PIP}_{2}\right)$ depletion following SILP exposure in neurons. ${ }^{6} \mathrm{PIP}_{2}$ depletion is involved in modulation of the neuronal KCNQ potassium channels, which initiate AP stimulation. ${ }^{6,20-22}$ Alternatively, sustained high concentrations of calcium, delayed $\mathrm{PIP}_{2}$ recovery, and disrupted membrane integrity may prevent the cell from returning to the resting potential and effectively prevent APs, ${ }^{6}$ leading to the AP block observed here.

As demonstrated, INI provides an alternative means of AP block in neurons. The advantages of INI include precise and selective illumination by fiber or free beam optics, direct contact is not necessary, and exposure can be precisely tuned to vary AP block duration. Optogenetic means of AP block, such as with halorhodopsins and yellow light, offer the same selectivity in a noncontact manner. However, optogenetics requires genetic manipulation, limiting its potential applications. SILPs provide a robust platform for both AP stimulation and block without genetic modification, and therefore, may be instrumental for studies seeking to identify, observe or control neural circuitry, or for biotechnologies seeking to interface directly with natural nervous systems, such as for neural-integrated prosthetics.

\section{Disclosures}

The authors have no additional relevant financial interests or potential conflicts of interest.

\section{Acknowledgments}

This work was supported by grants from the Air Force Office of Scientific Research (LRIR \#15RHCOR204 and LRIR \#14RH02COR). Dr. Walsh was supported by a fellowship from the National Research Council. FCK-Optopatch2 was a gift from Adam Cohen (Addgene plasmid \#51694). The authors thank Chad Oian for assistance with the SESE model simulations.

\section{References}

1. J. Wells et al., "Optical stimulation of neural tissue in vivo," Opt. Lett. 30(5), 504-506 (2005).

2. J. Wells et al., "Application of infrared light for in vivo neural stimulation," J. Biomed. Opt. 10(6), 064003 (2005).

3. J. M. Cayce et al., "Infrared neural stimulation of primary visual cortex in non-human primates," Neuroimage 84, 181-190 (2014).

4. J. M. Cayce et al., "Pulsed infrared light alters neural activity in rat somatosensory cortex in vivo," Neuroimage 57(1), 155-166 (2011).

5. J. Wells et al., "Biophysical mechanisms of transient optical stimulation of peripheral nerve," Biophys. J. 93(7), 2567-2580 (2007).

6. H. T. Beier et al., "Plasma membrane nanoporation as a possible mechanism behind infrared excitation of cells," J. Neural Eng. 11(6), 066006 (2014).

7. E. S. Albert et al., "TRPV4 channels mediate the infrared laser-evoked response in sensory neurons," J. Neurophysiol. 107(12), 3227-3234 (2012).

8. A. R. Duke et al., "Transient and selective suppression of neural activity with infrared light," Sci. Rep. 3, 2600 (2013).

9. H. J. Feng et al., "Alteration of GABAergic neurotransmission by pulsed infrared laser stimulation," J. Neurosci. Methods 192(1), 110-114 (2010).

10. N. Bhadra and K. L. Kilgore, "Direct current electrical conduction block of peripheral nerve," IEEE Trans. Neural Syst. Rehabil. Eng. 12(3), 313-324 (2004)

11. C. Tai, W. C. De Groat, and J. R. Roppolo, "Simulation analysis of conduction block in unmyelinated axons induced by high-frequency biphasic electrical currents," IEEE Trans. Biomed. Eng. 52(7), 1323-1332 (2005).

12. P. Seeman, "The membrane actions of anesthetics and tranquilizers," Pharmacol. Rev. 24(4), 583-655 (1972).

13. K. Kaneda et al., "Selective optical control of synaptic transmission in the subcortical visual pathway by activation of viral vector-expressed halorhodopsin," PLoS One 6(4), e18452 (2011).

14. R. S. McIsaac, C. N. Bedbrook, and F. H. Arnold, "Recent advances in engineering microbial rhodopsins for optogenetics," Curr. Opin. Struct. Biol. 33, 8-15 (2015).

15. M. G. Shapiro et al., "Infrared light excites cells by changing their electrical capacitance," Nat. Commun. 3, 736 (2012).

16. W. G. A. Brown et al., "Whole cell patch clamp for investigating the mechanisms of infrared neural stimulation," J. Visualized Exp. 77, e50444 (2013).

17. D. R. Hochbaum et al., "All-optical electrophysiology in mammalian neurons using engineered microbial rhodopsins," Nat. Methods 11(8), 825-833 (2014).

18. J. M. Kralj et al., "Optical recording of action potentials in mammalian neurons using a microbial rhodopsin," Nat. Methods 9(1), 90-95 (2012).

19. C.-P. Richter et al., "Neural stimulation with optical radiation," Laser Photonics Rev. 5(1), 68-80 (2011).

20. G. P. Tolstykh et al., "Activation of intracellular phosphoinositide signaling after a single 600 nanosecond electric pulse," Bioelectrochemistry 94, 23-29 (2013).

21. L. F. Horowitz et al., "Phospholipase $\mathrm{C}$ in living cells: activation, inhibition, $\mathrm{Ca}^{2+}$ requirement, and regulation of M current," J. Gen. Physiol. 126(3), 243-262 (2005).

22. P. Delmas and D. A. Brown, "Pathways modulating neural KCNQ/M (Kv7) potassium channels," Nat. Rev. Neurosci. 6(11), 850-862 (2005). 